\title{
A Semantic Web Solution for Enhancing The Interoperability of E-learning Systems by Using Next Generation of SCORM Specifications
}

\author{
https://doi.org/10.3991/ijet.v14i11.10342 \\ Abdellah Bakhouyi $\left.{ }^{(}\right)$, Rachid Dehbi, Mouad Banane, Mohamed Talea \\ Hassan II University of Casablanca, Casablanca, Morocco \\ abdellah.bakhouyi@gmail.com
}

\begin{abstract}
E-learning is a very dynamic field, constantly growing, which refers to an educational content or learning experiences provided or made by means of digital technologies. The development of this area has a direct impact on the quality of education and cost reduction.

E-learning is now dominated by learning management systems such as Moodle, to improve the interoperability of these systems; the use of data / resources is a major requirement, this need to push the e-Learning community to seek new approaches to improve this interoperability of systems. On the other hand, semantic Web technologies offers standards like RDF allows to describe Web resources with their metadata. We propose in this work an approach based on Semantic Web technology to improve the interoperability of e-Learning systems like Moodle.
\end{abstract}

Keywords-Semantic web, RDF, E-learning, SCORM, interoperability, LRS, LMS, Moodle

\section{Introduction}

In recent years, the field of interoperability of e-Learning systems had several successive evolutions. Interoperability is mainly about allowing heterogeneous information systems to work together to achieve a common goal. Initially, interoperability was to build distributed or federated architectures where the main challenge was to translate the data patterns of a source model into a target model. The interoperability locks were numerous, we can cite the main ones such as syntactic heterogeneity, schematic heterogeneity or the most recent, semantic heterogeneity. Each of these locks has been the subject of numerous studies proposing architectures and tools [1][2][3] which go from specific translators between pairs of models to the design of metamodels, canonical models or supermodels to federate all the resources in a global model. Each cycle of research on these locks has ended on the adoption by the community of a standard or standard of knowledge representation in this area of online learning. One can quote the definition of TCP / IP for the resolution of the problems of heterogeneity of the protocols of exchanges on networks [4], the definition of the 
XSD standard to address schematic heterogeneity problems or the XML standard to address syntactic heterogeneity problems [5]. The only shadow in this cycle of standard adoption solutions is semantic heterogeneity. Semantics can be defined in this area as the meaning of knowledge [6]. Semantic heterogeneity thus concerns a divergence of understanding of an element that can be represented in a unique and common way by cooperating information systems. This divergence of understanding leads to divergences of treatment. Cooperation is quickly limited. The definition also of semantic Web standards such as RDF and RDF (S) can be used to answer the problems of syntactic and semantic heterogeneity of E-learning systems.

To address the semantic interoperability problem of e-learning systems or other area, the community has set new standards. Semantic languages composed of operators modeling and manipulating semantic graphs. These languages, such as RDF, form the basis of what is currently called the semantic web. The semantic web aims to develop a smart web where information is understood by machines. Thus, the notion of metamodel and the notion of ontology. The ontology, initially defined in the field of philosophy as: the study of being as being, represents, in our field of interoperability, a model of representation of the meaning of the knowledge of a field [6].

To answer our turn to these locks, we wanted to deal with this problem of interoperability of e-learning systems from a new angle still untapped in the field of elearning. We wanted to establish a high quality of communication and sharing between the LRS and the CDN, or between the LRS and the LMS system to apply them to the management and qualification of semantic data.

Our main contributions in this work are:

- The improvement of interoperability between e-Learning systems and in particular their mobile extension using / based on RDF.

- The transformation of the file stored in the LRS (Learning Record Store) system containing statements comes from the LMS system, this JSON file will be transformed into RDF using the JSON-LD format [7].

\section{Semantic Web and E-Learning Standards for Interoperability}

The standards of content and online learning structure are established to ensure the interoperability of e-learning systems so that access to sources of information such as reuse of content or discrimination of subjects from different sources at different times is possible [8]. Standards such as Advanced Distributed Learning (ADL SCORM [9]) have been widely implemented and adopted, so that standards have been implemented to provide data models and communication protocols conforming to have an interoperable content between e-learning systems.

When these standards are incorporated into online learning systems, users can create and use content from multiple systems from different vendors, knowing that they will work effectively together based on their quality and relevance [9]. One of the great challenges of e-learning systems is the exchange of data between different systems. 
Recent Developments in e-learning interoperability standards, learning design and other research on the pedagogy of semantic e-learning have presented an inclination of Semantic Web technologies to encourage learning services focused on the learning content aware services and personalized [10-12]. Technologies based on ontologies and intelligent agents should support the processing of semantic information on the Semantic Web [13]. Most learning management system are currently working in a closed system. Some systems still use their own framework for learning the description of the content, instead of adopting the interoperability standard. The concept of course, student, pedagogical resource, summary or class must be formally described to be shared between all the systems of an educational institution [10-13].

These interoperability issues affect the flexibility of the teaching-learning process and result in decreased end-user satisfaction and learning success.

\subsection{Next generation of SCORM: XAPI and CMI5 specifications}

In its development, the SCORM standard [9] is characterized by the use of XML technology, to define the structure of the course in its first versions as well as the use of metadata to determine the details of the content, while the SCORM specification 2004 and in particular, brought two more important aspects to avoid any ambiguity in later versions; Navigation and sequencing to verify compliance and interoperability in learning environments [14].

The evolution of SCORM has been extremely positive and does not end in the 2004 version [14], but it lacks good practices in terms of the means and mechanisms for exploiting tracing data and tracking learning experiences and does not support the features and services that learners can take their courses in mobile applications.

With the technological development of interoperability standards, portals and learning systems and applications need to track their learning experiences and activities. Tin Can API is an open source standard, recently modernized to replace SCORM (Sharable Content Object Reference Model). In 2013, the third version of Tin Can API formalized under the name Experience API or xAPI and part of the Training Learning Architecture (TLA) [15]. It is a Representational state transfer (REST) web service based on JavaScript Object Notation (JSON) for its data format. XAPI is a specification that produced an application program interface (API), which captures the learning data in the stream and stores them in a repository in the form of "statement" objects using the following structure:

<actor> <verb> <object>, with <result>, in <context> 


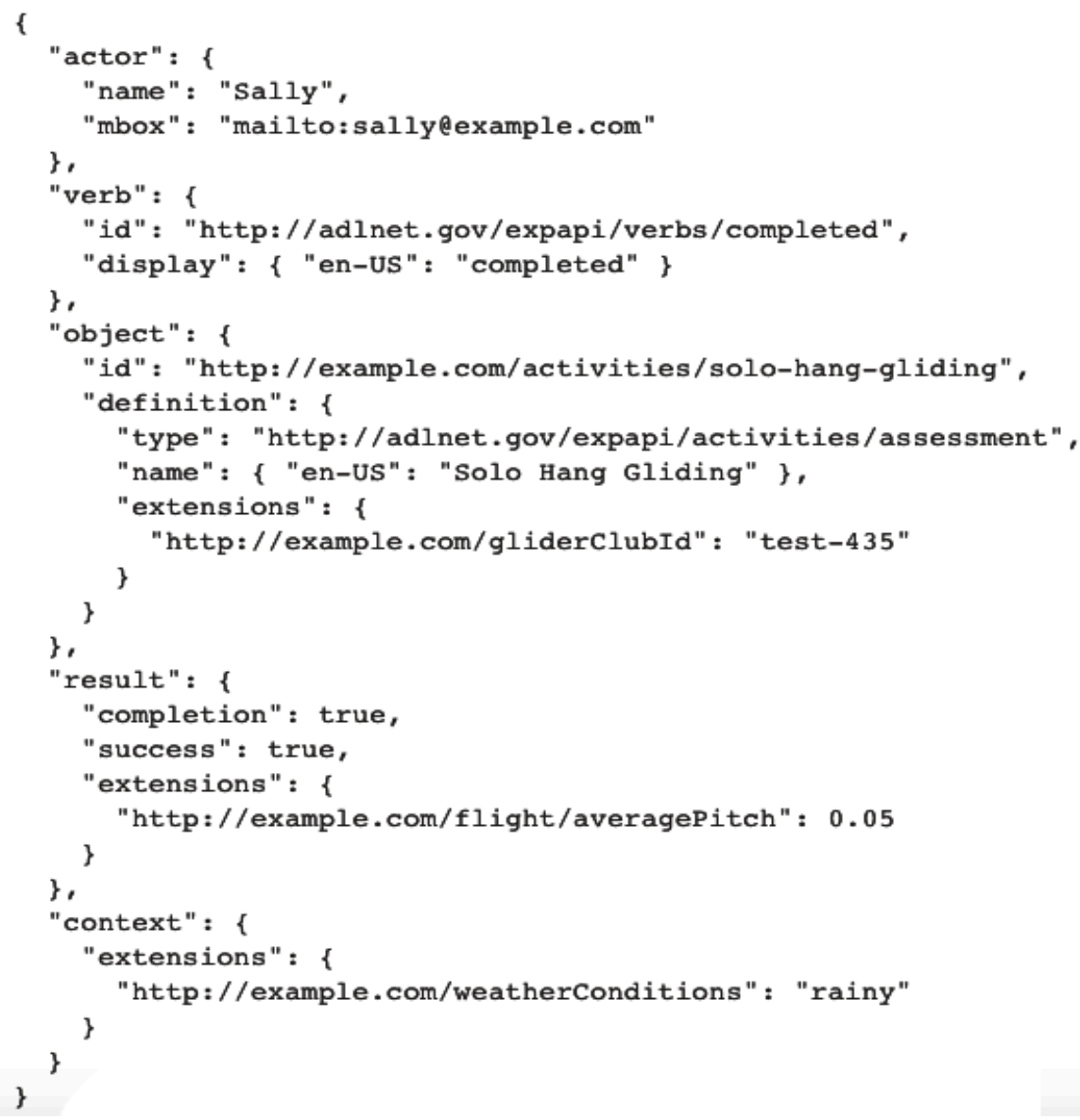

Fig. 1. Representation JSON of a XAPI statement.

xAPI is selected from the reviewed and compared specifications xAPI, ActivityStrea.ms [16], IMS ${ }^{1}$ Caliper [17], $\mathrm{CAM}^{2}$ (which is an early approach to capturing and storing attention metadata for single users), NSDL paradata ${ }^{3}$ (defined to capture aggregated usage data about a resource which is designated by audience, subject or education level) and Organic.Edunet format [18] (to store social data provision activities and designed in an extendable way, so other user activities) for communication between heterogeneous learning environments in a major contribution that was published [19].

CMI-5 [14] is a set of rules with the use of xapi protocol (or profile xapi) which is intended for scenarios launch contained within a learning management system. It is

${ }^{[1]}$ IMS Global Learning Consortium

${ }^{[2]}$ Contextualized Attention Metadata

${ }^{[3]}$ National Science Digital Library Paradata 
indispensable to know that XAPI as it is does not replace SCORM, because it is only responsible to match the learning experience with an LRS (learning record score) to LMS. In addition, the set of rules includes some verbs: Launched, Initialized, Completed, Passed, failed, Abandoned, and Terminated. They are used to match the learning content (AU) with an LMS, and correlate its data with the Learning Record Store (LRS), which is considered as a communication path between the Learning Management System (LMS) and the $\mathrm{AU}^{4}$ (Fig. 2).

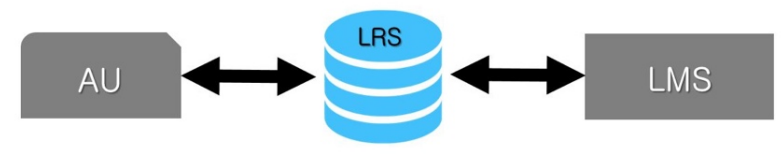

Fig. 2. CMI5 Communication Paths

\subsection{Semantic web in E-learning}

Some studies have been conducted to verify that the LMS is complete in university [20][21][22]. Previous research used the concept of synchronization between learning media in LMS. However, students can not attend classes at another university. Only the administrator can synchronize. On the other hand, lifelong learning networks must be able to connect some LMSs without any access restrictions for users.

Semantic Web technology and the use of ontologies are able to provide the computer semantics and interoperability required for task automation in the learning management system [21].

The Semantic Web is a set of standards, data structures and software that enhance the online experience in a more detailed and intelligent way [22]. The Semantic Web offers students the ability to get rich content related content on their desktop without identifying or explicitly asking for it. However, studies on the interoperability of learning management systems in the Semantic Web have not been fully exploited.

The main model of our Semantic Web research is layered architecture. The layered architecture comprises a number of hierarchically organized layers, as in Fig. 3. It can take many forms, such as the pyramid shape, the circular shape and the shape of the tower, as in the Semantic Web. Each layer provides a service to its top layer and serves as a client to the lower layer. This type of architecture depends on the sequence of incremental steps, which means an increasing level of abstraction: if the function of a layer changes, it means that two other layers can be affected. The most famous example of layered architecture is ISO (International Organization for Standardization) and OSI (Interconnected Open Systems) [23].

The four semantic Web architecture versions created by Tim Berners-Lee are explained in many research articles. To do this, we present and explain only the latest version that consists of four layers are needed, as shown in Fig 3.

${ }^{[4]}$ Assignable Unit (Data) 


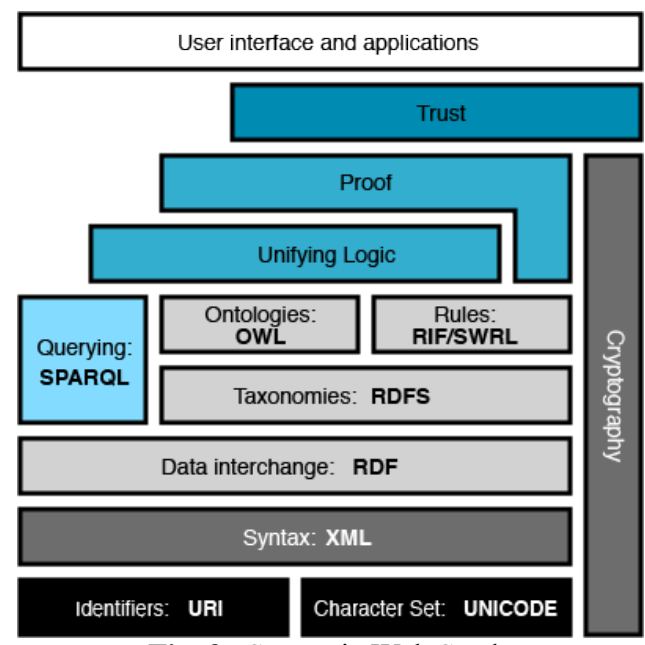

Fig. 3. Semantic Web Stack

- The XML layer, which represents the data

- The RDF layer, which represents the meaning of the data

- The ontology layer, which represents the formal common agreement on the meaning of the data

- The logical layer, which allows intelligent reasoning with meaningful data

The Semantic Web is an extension of the current web in which information is associated with a well-defined meaning that improves the ability of computers and people to work cooperatively. The purpose of this Web, also known as Web 3.0 [23], is to describe a method for exposing, sharing and connecting data via URIs on the WEB. (A URI, Uniform Resource Identifier, is a short string of characters that identifies a resource on a network)

As a result, our efforts must be focused on improving existing indexing tools, while focusing on the acquisition of new data based on RDF schemas.

\section{A Semantic Web Solution to Enhance The Interoperability of E-learning Systems}

\subsection{Approach}

Our approach is to ensure better semantic interoperability at the LRS to store the interactions that come from the LMS, the use of RDF with its structure of Triple (Subject, predicate, object) offers a better management of traceability of the LMS logs and also to ensure the high availability of these data, and a content interoperability improvement between learning systems mentioned above by using RDF / XML [24] is a 
very good means of communication and information sharing. For Web resources: Courses, Image, Document etc., are identified by a unique URI.

This approach with their features will apply on our web service architecture (Fig. 4) that was previously designed based on the next generation of SCORM [25] to improve the interoperability of e-learning content.

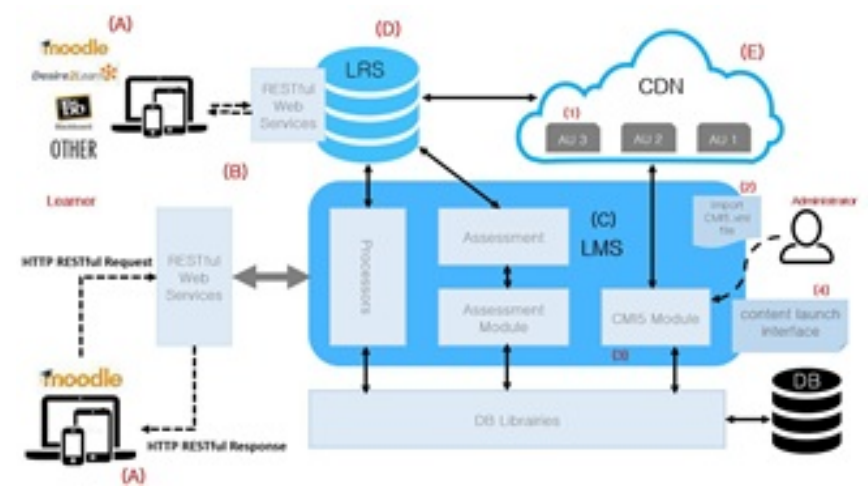

Fig. 4. SOA architecture for interoperability of LMS and mobile applications [25]

\subsection{JSONtoRDF TransLRS system}

To transform our JSON file into an RDF document, we can use a mapping tool named JSON-LD [26]. After adding JSON-LD context to JSON data, we can easily reinterpret this data as an RDF document that contains RDF triples, the principle of this context is the mapping of JSON attributes into RDF properties. The figure Fig. 5 demonstrates the transformation architecture from JSON to RDF document using the JSON-LD mapping tool

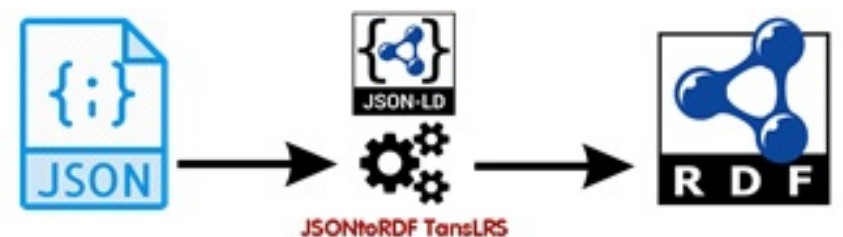

Fig. 5. JSON2RDF TansLRS architecture

In this paper, we present a conversion module called JSON2RDF TransLRS, with the goal of transforming JSON formatted statements into an RDF document at the heart of the LRS system. We tried to transform an example of a video statement from the LMS into JSON format. The figure (Fig .6) shows the JSON2RDF TransLRS tool window. 
Paper-A Semantic Web Solution for Enhancing The Interoperability of E-learning Systems by Using ...

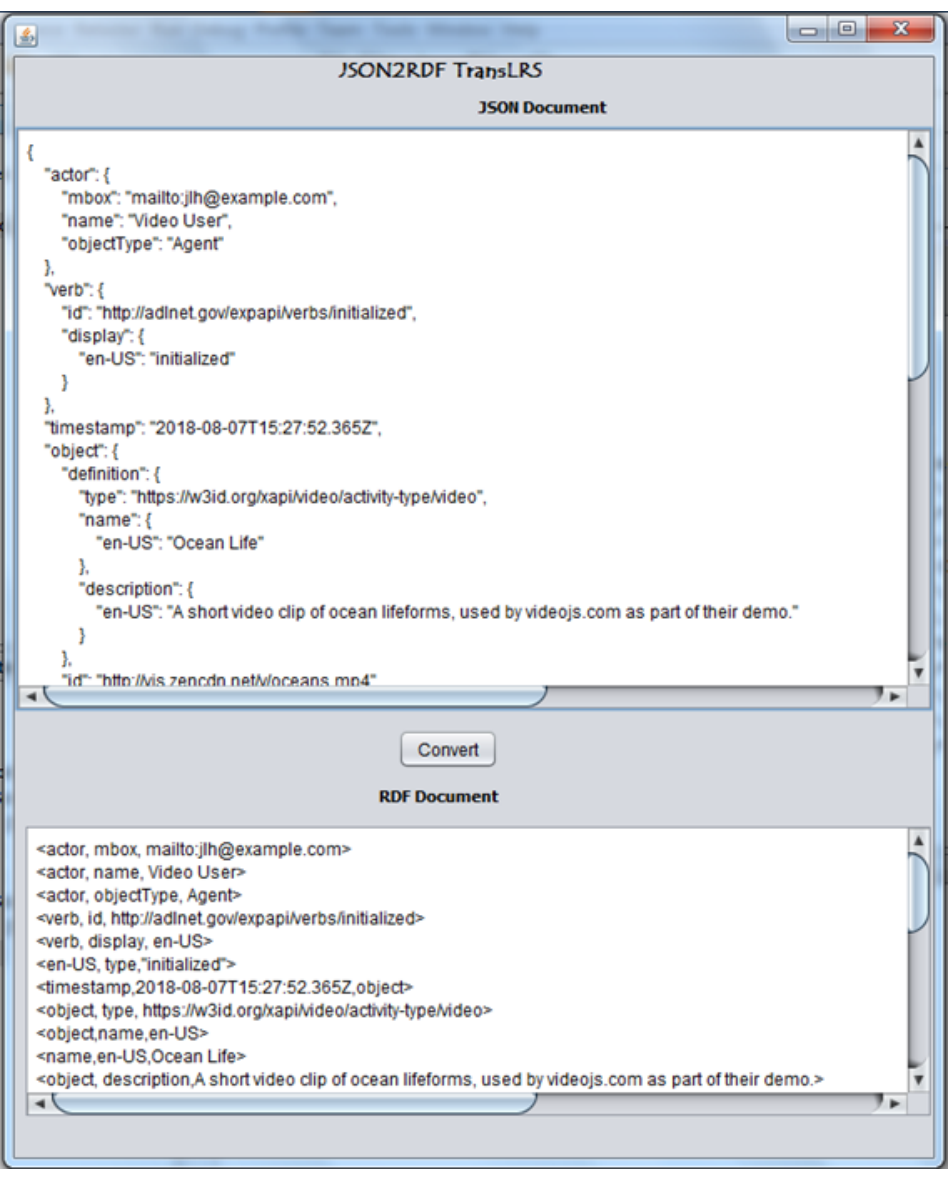

Fig. 6. Screenshot of the JSON2RDF TransLRS tool window

The following figure (Fig. 7) shows the JSON2RDF TransLRS transformation program developed in Java and thanks to the object-oriented functionality, we can allow to create modular programs (dividing a program into separate subroutines) and reusable code and also the functionality platform independent that allows us to easily switch from one computer system to another. 


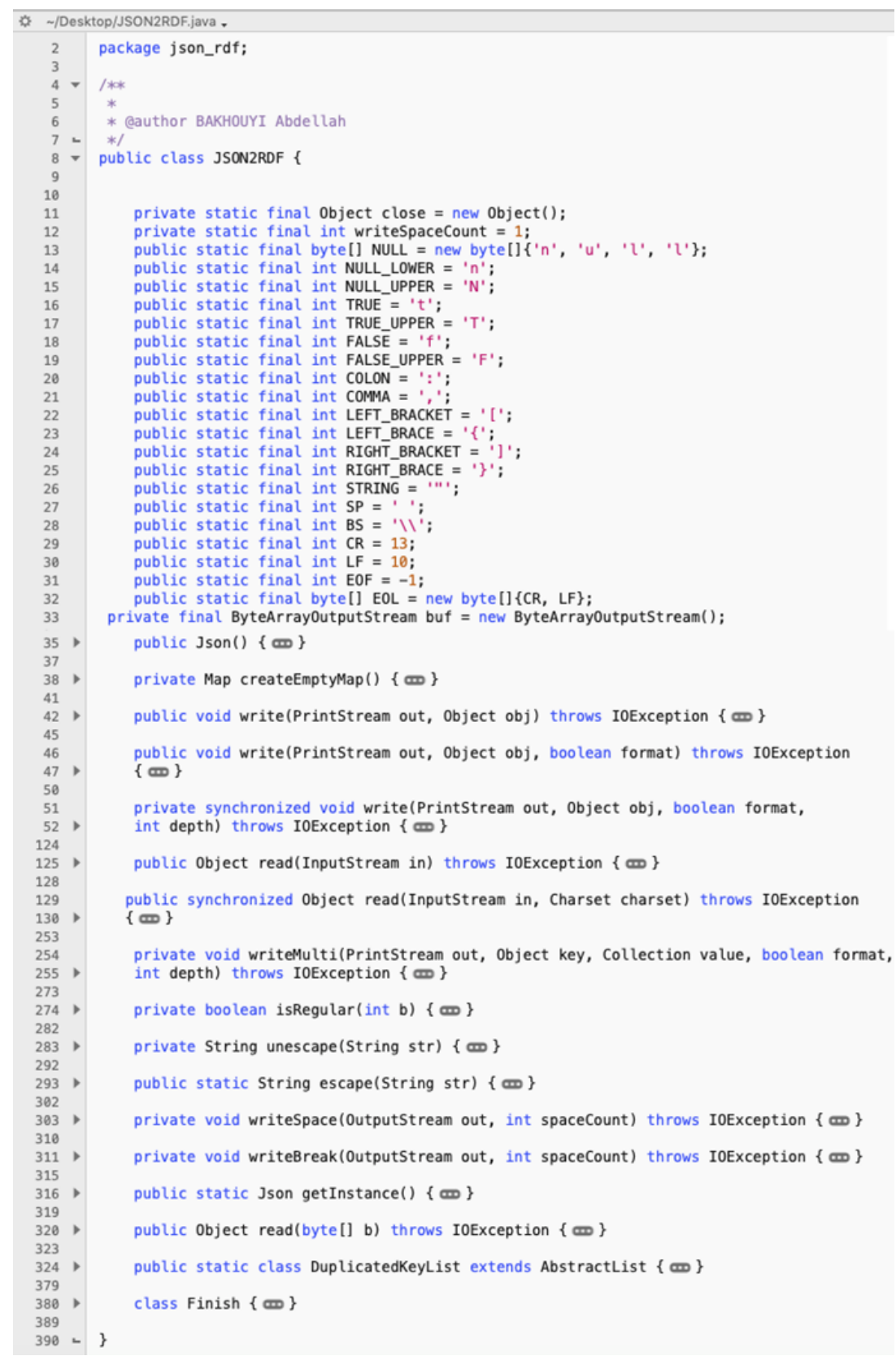

Fig. 7. Screenshot of the JSON2RDF TransLRS program summary 


\section{Conclusion}

Improving interoperability between e-learning systems and their mobile extension is really needed. It will help users access the content and features of the learning management system via mobile devices. According to this article, content standards have a very effective role in ensuring the interoperability of learning systems and, in particular, most standards have an important perspective including the next generation of SCORM. On the other hand, it is necessary to base on a very intelligent standard which has not been generally exploited, it is the Semantic Web to have a better interoperability of the management systems of the more robust learning. For the reasons mentioned above, This article aimed at designing a Semantic Web technology-based approach that aims to transform JSON format declarations into RDF format by using the JSON-LD mapping tool, which statements come from the LMS and store in the LRS system to improve the interoperability of e-learning systems such as Moodle and their mobile extension. In particular, we took the case of videos as type of activity in the statements.

We have seen that this architecture will always be in development and in perspective. In the future, more research is needed using semantic interoperability to further develop e-learning systems and their mobile extensions by integrating new technologies that will enable learners to easily access to the gigantic learning resources learning, as well as helping teachers and administrators to perform statistics and reportsfrom no-SQL data and integration object smaller than the mobile (Internet of Things IoT).

\section{$5 \quad$ References}

[1] Berners-Lee T. (2000). What the semantic web can represent, http://www.w3.org/DesignIssues/RDFnot.html

[2] Vdovjak, R., \& Houben, G. J. (2001, April). RDF-Based Architecture for Semantic Integration of Heterogeneous Information Sources. In Workshop on information integration on the Web (pp. 51-57).

[3] El-Seoud, S. A., El-Sofany, H., \& Karam, O. (2015). Semantic Web Architecture and its Impact on E-learning Systems Development. International Journal of Emerging Technologies in Learning (iJET), 10(5), 29-34. https://doi.org/10.3991/ijet.v10i5.4754

[4] Barakat, C., Altman, E., \& Dabbous, W. (2000). On TCP performance in a heterogeneous network: a survey. IEEE Communications Magazine, 38(1), 40-46. https://doi.org/10.1109/35.815451

[5] Nagarajan, M., Verma, K., Sheth, A. P., Miller, J., \& Lathem, J. (2006, September). Semantic interoperability of web services-challenges and experiences. In Web Services, 2006. ICWS'06. International Conference on (pp. 373-382). IEEE. https://doi.org/10.1109/icws.2006.116

[6] Berners-Lee, T., Hendler, J., \& Lassila, O. (2001). The semantic web. Scientific american, 284(5), 34-43. https://doi.org/10.1038/scientificamerican0501-34

[7] Sporny, M., Longley, D., Kellogg, G., Lanthaler, M., \& Lindström, N. (2014). JSON-LD 1.0. W3C Recommendation, 16, 41. 
Paper-A Semantic Web Solution for Enhancing The Interoperability of E-learning Systems by Using ...

[8] Fallon, C., \& Brown, S. (2016). E-learning standards: a guide to purchasing, developing, and deploying standards-conformant e-learning. CRC Press.

https://doi.org/10.1201/9781420025330

[9] Del Blanco, Á., Serrano, Á., Freire, M., Martínez-Ortiz, I., \& Fernández-Manjón, B. (2013, March). E-Learning standards and learning analytics.

https://doi.org/10.1109/educon.2013.6530268

[10] Tiropanis, T., Davis, H., Millard, D., \& Weal, M. (2009). Semantic technologies for learning and teaching in the Web 2.0 era. IEEE Intelligent Systems, 24(6), 49-53. https://doi.org/10.1109/mis.2009.121

[11] Garcia-Gonzalez, H., Gayo, J. E. L., \& Paule-Ruiz, M. (2017). Enhancing e-Learning content by using Semantic Web technologies. IEEE Transactions on Learning Technologies, 10(4), 544-550. https://doi.org/10.1109/tlt.2016.2629475

[12] Tsortanidou, X., Karagiannidis, C., \& Koumpis, A. (2017). Adaptive educational hypermedia systems based on learning styles: The case of adaptation rules. International Journal of Emerging Technologies in Learning (iJET), 12(05), 150-168. https://doi.org/10.3991/ijet.v12i05.6967

[13] Manickasankari, N., Arivazhagan, D., \& Vennila, G. (2014). Ontology based Semantic Web technologies in E-learning environment using protege. Indian Journal of Science and Technology, 7(S6), 64-67.

[14] Bakhouyi, A., Dehbi, R., Lti, M. T., \& Hajoui, O. (2017, July). Evolution of standardization and interoperability on E-learning systems: An overview. In Information Technology Based Higher Education and Training (ITHET), 2017 16th International Conference on (pp. 1-8). IEEE. https://doi.org/10.1109/ithet.2017.8067789

[15] Total Learning Architecture - ADL Net. Retrieved from https:/www.adlnet.gov/tla/

[16] Vozniuk, A., Govaerts, S., \& Gillet, D. (2013, July). Towards portable learning analytics dashboards. In Advanced Learning Technologies (ICALT), 2013 IEEE 13th International Conference on (pp. 412-416). IEEE. https://doi.org/10.1109/icalt.2013.126

[17] Sakurai, Y. (2014, June). The value improvement in education service by grasping the value acceptance state with ict utilized education environment. In International Conference on Human Interface and the Management of Information (pp. 90-98). Springer, Cham. https://doi.org/10.1007/978-3-319-07863-2 10

[18] Niemann, K., Wolpers, M., Stoitsis, G., Chinis, G., \& Manouselis, N. (2013, April). Aggregating social and usage datasets for learning analytics: Data-oriented challenges. In Proceedings of the Third International Conference on Learning Analytics and Knowledge (pp. 245-249). ACM. https://doi.org/10.1145/2460296.2460345

[19] Wang, H. (2017). Construction of xAPI-based Multimedia Interaction Technology in Architectural Design Teaching. International Journal of Emerging Technologies in Learning (iJET), 12(07), 101-111. https://doi.org/10.3991/ijet.v12i07.7220

[20] Ford C, Mcnally D and Ford K 2017 Understanding Faculty Use of the Learning Management System Online Learn Consort J pp 50-67

[21] Masud, M. (2016). Collaborative e-learning systems using semantic data interoperability. Computers in Human Behavior, 61, 127-135. https://doi.org/10.1016/j.chb.2016.02.094

[22] Halimi, K., Seridi-Bouchelaghem, H., \& Faron-Zucker, C. (2014). An enhanced personal learning environment using social semantic web technologies. Interactive Learning Environments, 22(2), 165-187. https://doi.org/10.1080/10494820.2013.788032

[23] Zimmermann, H. (1980). OSI reference model--The ISO model of architecture for open systems interconnection. IEEE Transactions on communications, 28(4), 425-432. https://doi.org/10.1109/tcom.1980.1094702 
[24] Aghaei, S., Nematbakhsh, M. A., \& Farsani, H. K. (2012). Evolution of the world wide web: From WEB 1.0 TO WEB 4.0. International Journal of Web \& Semantic Technology, 3(1), 1. https://doi.org/10.5121/ijwest.2012.3101

[25] Bakhouyi, A., Dehbi, R. \& Talea, M. (2018). A New Web Service Architecture for Enhancing the Interoperability of LMS and Mobile Applications Using the Next Generation of SCORM. In International Conference on Advanced Intelligent Systems for Sustainable Development AI2SD 2018, AISC 915, pp. 1-8, 2019. https://doi.org/10.1007/978-3-03011928-7 65

[26] World Wide Web Consortium. (2014). JSON-LD 1.0: a JSON-based serialization for linked data.

\section{Authors}

Abdellah Bakhouyi is a PhD at the Faculty of Sciences Ben M'Sik, Laboratory of Information Processing (LIP), Hassan II University of Casablanca, Morocco. His research fields: E-learning, M-learning, Interoperability of E-learning Systems, Semantic Web, Model Driven Engineering, Big Data and Analytics and Internet of Thing.

Rachid Dehbi is an associate professor at the Faculty of Science Aïn Chock, and member of LR2I laboratory (Research laboratory in Computer Innovation). He is responsible of research strategies and doctoral education training process at Hassan II University of Casablanca. His work focuses on E-learning, M-learning, E-health, Mhealth, Model Driven Engineering, Database Security, Big Data,and Internet of Thing.

Mouad Banane is a PhD at the Faculty of Sciences Ben M'Sik, Laboratory of Information Technology and Modeling (LTIM), Hassan II University of Casablanca, Morocco. His research fields: Model Driven Engineering, Semantic Web, Big Data and Analytics and Internet of Thing.

Mohamed Talea is a professor of higher education at the Faculty of Sciences Ben M'Sik and Research Director of the Laboratory of Information Processing (LIP), Hassan II University of Casablanca, Morocco. His major research interest is on Systems engineering, Networking and security of information systems.

Article submitted 2019-02-19. Resubmitted 2019-04-03. Final acceptance 2019-04-04. Final version published as submitted by the authors. 